\title{
Sublingual Swelling - A Diagnostic Dilemma
}

\author{
VU Shanmugam ${ }^{1}$, Vidyachal Ravindra ${ }^{1 *}$, RutaShanmugam ${ }^{1}$, RG Mariappan ${ }^{1}$, Balaji Swaminathan ${ }^{1}$, Prem \\ Nivas $^{1}$, C Dhanashekaran ${ }^{2}$ and SK Srinivasan ${ }^{2}$ \\ ${ }^{1}$ Department of ENT, RMMCH, Annamalai University, India \\ ${ }^{2}$ Department of Anesthesia, RMMCH, Annamalai University, India
}

Submission: November 23, 2016; Published: December 09, 2016

\begin{abstract}
We report a rare case of sublingual swelling in an 11 month old child which was a diagnostic and surgical challenge. The child presented with a painless, bluish, and swelling in the sublingual and submental region with complaints of protrusion of tongue. A pre-operative CT scan was done, but a diagnosis was not truly established. Though it was a case of difficult intubation, surgical excision was done. During surgery Ranula was suspected but histopathological examination revealed it to be Vascular Hamartoma masquerading as a Ranula. Although rare, vascular malformation should be part of differential diagnosis in sublingual and submental swellings.
\end{abstract}

Keywords: Sublingual swelling; Vascular hamartoma; Hemangioma; Lymphangioma

\section{Introduction}

Hamartoma is derived from the Greek word hamartia meaning fault or defect and -oma denoting tumor. It was coined by Albrecht in 1904 to denote developmental tumorlike malformation [1]. It is defined as a non neoplastic developmental malformation, comprising of normal mature cells which are native to the anatomic location [1]. Histology shows disorganized architectural pattern with predominance of one of its components. They are common in lung, pancreas, spleen, liver and kidney but very rare in the head and neck region. It is commonly asymptomatic. But morbidity can arise due to obstruction, infection, infarction, hemorrhage, and rarely due to neoplastic transformation. Deeper masses such as the mass described in this present case, can cause Respiratory and swallowing disturbance. It may occasionally cause life threatening respiratory distress. Differential diagnostics of neck masses is a challenge. Initially the firm sublingual swelling was assumed to be Pleomorphic Adenoma. But intra-operatively the mass resembled a large Ranula. Post-operative histopathological examination revealed it to be Vascular Hamartoma.

\section{Case Report}

We report a rare case of Vascular Hamartoma of the Sublingual Region masquerading as a Ranula. An 11 month old child presented to the ENT OPD with complaints of sublingual swelling of 2 months duration with protrusion of tongue since childhood which was ignored by the parents. Presently, the obstructive nature of the swelling has led to the child to develop difficulty in both respiration and swallowing, which has prompted the parents to come to the hospital. Bimanual palpation revealed a swelling in the floor of the mouth, $3 \times 3$ $\mathrm{cm}$ in size, ovoid in shape, bluish in colour, non-tender and firm in consistency. A provisional differential diagnosis of Ranula, Hemangioma, Lymphangioma, Pleomorphic Adenoma and Congenital Dermoid Cyst was made.

\section{Pre 0perative}

USG Neck: Multi-loculated cystic lesion in Submental Region. Differential Diagnosis - Lymph Cystic Lesion or Plunging Ranula.

CT - Neck: Hypodense lesion below the mandible in midline displacing the Genioglossus muscle. Extending into the submandibular space. Mylohyoid muscle was pushed down.

The risk of life threatening respiratory distress made surgery necessary. Thus exploration on table with excision was planned under general anesthesia. It was a case of difficult intubation with standby Tracheostomy. Through an external neck incision, swelling was identified on retracting the mylohyoid muscle. Swelling was dissected from its surrounding tissue. Extension into the substance of the tongue was noted. The swelling was removed in toto. Corrugated drain was kept in site. Airway was maintained with Nasopharyngeal airway and Ryle's tube was placed (Figures 1 \& 2). 


\section{Global Journal of Otolaryngology}

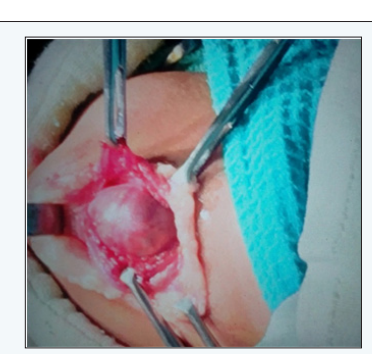

Figure 1: Exposure of the Sublingual Mass following retraction of Mylohyoid.

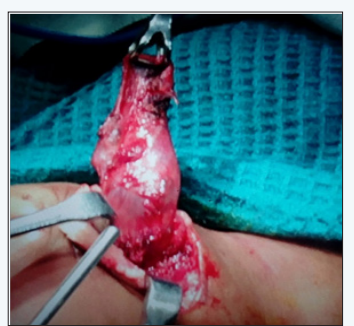

Figure 2: Following dissection of the mass from the surrounding tissue.

\section{Post-Operative:}

Histopathology: Ciliated vascular channels surrounded by fibro-collagenous tissue and skeletal muscle tissue. Final histopathological diagnosis was a Vascular Hamartoma.

Post-operative the child was uneventful and the child was symptomatically better. Nasopharyngeal Airway was removed on the third post-operative day. Ryle's tube was removed on the 6th post-operative day. Residual tongue edema remained. Protrusion of tongue drastically reduced. The floor of mouth swelling resolved and the cervical wound healed normally (Figures $3 \& 4$ ).

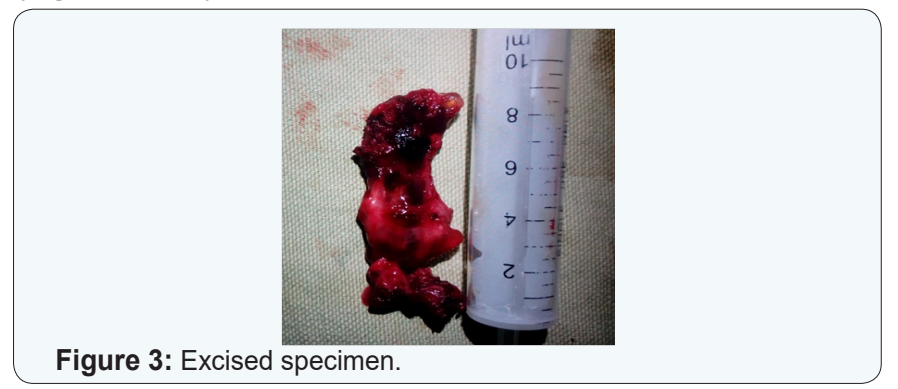

Figure 3: Excised specimen.

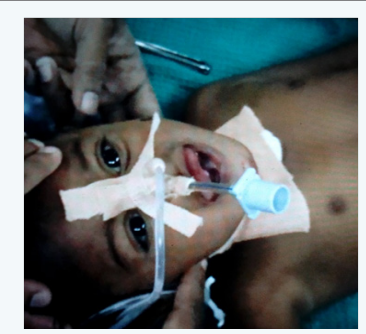

Figure 4: Post-operative picture with Nasal Airway and Ryle's Tube.

\section{Discussion}

Vascular Hamartomas are a non-neoplastic abnormality that occurs due to errors in vascular morphogenesis. During organogenesis, abnormalities in the regulating factors can lead to improper proportion and impairment in differentiation [2]. Thus defect in the regulatory pathway of vascular stem cells leads to formation of hamartomatous lesion [2]. They may occur as primary lesion or in association with syndromes such as Sturge-Weber, Klippel-Trenaunary, Proteus Syndrome, Bannayan-Riley-Ruvalcaba Syndrome and Osler-Weber-Rendu Syndrome. Lymphangioma is a congenital Hamartoma involving the lymphatic system. They are common in the head and neck region but very rare in the oral cavity. They were first described for the first time by Redenbacher in 1828 [3].

In patients less than 20 years of age, they represent $6 \%$ of all benign tumors of smooth tissue [3]. Around 50\% are noted at birth and around $90 \%$ develop by the age 2 years. Clinically, lymphangiomas of the oral cavity present with a plaque made up of small vesicles with thin walls. These vesicles may be filled with lymph or blood. In the case presented here, these plaques were observed on the lingual surface of the tongue. Hamartoma are usually exophytic but may rarely present as a flat pigmented lesions. According to a study conducted by Kaplan, though Hamartoma of the oral cavity is very rare, it may occur on the tongue, labial mucosa, buccal mucosa, and median maxillary alveolus. One of the differential diagnosis that we suspected was a Plunging Ranula. Ranula is a extravasation cyst of the sublingual gland which represents $6 \%$ of all oral sialocysts [4].

Plunging Ranula has deep extension beyond the mylohyoid muscle. It can also have massive involvement of the submandibular and parapharyngeal spaces. Its clinical and radiological behavior can be misleadingly similar to other cystic neck masses, particularly the cystic hygroma. But while lymphoid malformations such as Lymphangiomas are present at birth or early childhood, ranulas typically appear in young adults. Both are centered in the submandibular space with a possible continuous extension beneath the free edge of the mylohyoid muscle. Whereas Plunging Ranulas involve only the parapharyngeal and sublingual spaces, lymphangiomas are far more infiltrative, extending further toward the para- and retropharyngeal, carotid, posterior cervical and visceral spaces and the mediastinum. In case of super-infection or previous surgery the radiological image of the Plunging Ranula can be deceptively similar to lymphangiomas.

In the past, the first line of management of vascular malformations which did not spontaneously involute was radical surgery. One of the disadvantages of radical surgery includes injury to muscle and nerves. Furthermore, in toto excision was only possible in about $40 \%$ of the cases and these anomalies have a high incidence of recurrence. Alternative managements like intra-cystic sclero-therapy can lead to disappointing cosmetic and functional result [2]. Presently OK-432 is the preferred intra-lesional sclerosant. It is a lyophilized mixture of low virulent Su Strain of type III group A Streptococcus pyogenes. When administered intra-lesionally it causes inflammation and infiltration with neutrophils and macrophages [5]. In the present 
case, surgical removal was chosen because of the increasing risk of life threatening respiratory distress and because the possibility of the diagnosis of a Plunging Ranula could not be excluded [6-10].

\section{Conclusion}

We report a case of Vascular Hamartoma of the sublingual region masquerading a Plunging Ranula. Although rare, vascular malformation should always be kept in mind in sublingual swellings.

\section{References}

1. Patil S, Rao RS, Majumdar B (2015) Hamartomas of the Oral Cavity. J Int Soc Prev Community Dent 5(5): 347-353.

2. Gabriella Kecskésa, László Rovóa, Péter Ragób, Márta Katonac, Szabolcs Tornyosc, et al. (2011) Respiratory distress caused by congenital mixed (lymphoid-venous) vascular hamartoma. International Journal of Pediatrics Otorhinolaryngology 6(4): 229-232.

3. Stănescu L, Georgescu EF, Simionescu C, Georgescu I (2006) Lymphangioma of Oral Cavity. Rom J Morphol Embryol 47(4): 373-377.

4. Abdul Bagi Mustafa, Kamran Bokhari, Master Luqman, Mohammad Shahul Hameed, Zaheer Kota (2013) Plunging Ranula: An Interesting Case Report. Open Journal of Stomatology 3(1): 118-121.
5. Rathan JJ, Vardhan BG, Muthu MS, Venkatachalapathy, Saraswathy K, et al. (2005) Oral Lymphangioma: A Case Report. J Indian Soc Pedod Prev Dent 23(4): 185-189.

6. Puneeta Vohra, Vinod Vijay Chandar, Ranjit Patil, Saumya Verma (2015) Cavernous hemangioma in the floor of oral cavity masquerading as a ranula. Journal of Indian Academy of Oral Medicine and Radiology 27(2): 286-290.

7. Arvinder Singh, Manjeet Kaur, Sohan Singh, Ramesh Chander, Suman Bhagat (2013) Giant Submental Hemangioma: A Rare Neck Mass. Journal of Evolution of Medical and Dental Sciences 2(48): 9415-9418.

8. Sharad Ramdas, Anita Ramdas, Moses M Ambroise, Renu G'Boy Varghese (2014) Submandibular vascular hamartoma with phleboliths mimicking sialolithiasis. Journal of Clinical Sciences 11(2): 52-54.

9. I Kaplan, I Allon, B Shlomi, V Raiser, S Kleinman (2015) A Comparative Study of Oral Hamartoma and Choristoma. Journal of Interdisciplinary Histopathology 3(4): 129-134.

10. Priscila Henriques Corrêa, Lara Cristina Caldeira Nunes, Aline Cristina Batista Rodrigues Johann, Maria Cássia Ferreira de Aguiar, Ricardo Santiago Gomez, et al. (2007) Prevalence of Oral Hemangioma, vascular malformation and varix in a Brazilian population. Braz Oral Res 21 (1): 40-45.

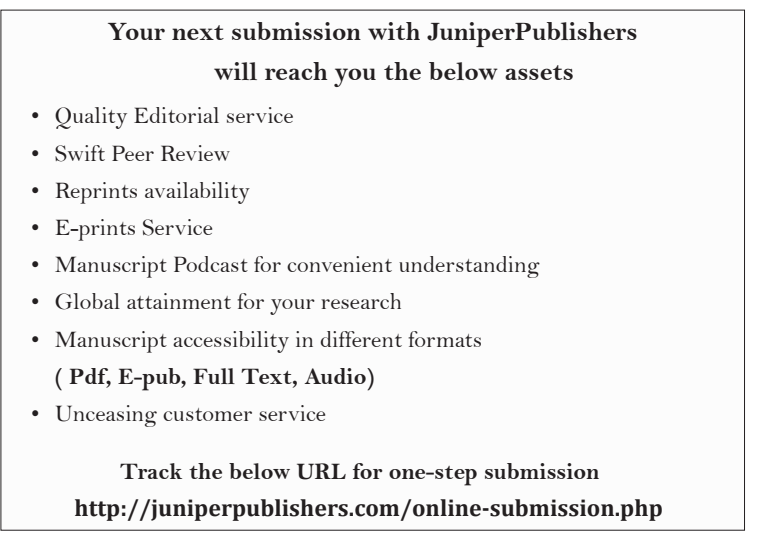

